\title{
Routine adult immunization in Canada: Recommendations and performance
}

\author{
BL Johnston MD FRCPC ${ }^{1}$, JM Conly MD CCFP FRCPC FACP ${ }^{2}$
}

\begin{abstract}
In May 2002, the Public Health Laboratory Service in England reported two cases of severe tetanus in women in their sixties (1). Both patients had documentation of receiving only one dose of tetanus toxoid (TT) in the past, one eight years previously and the other more than 20 years before, after an episode of tetanus. Also in May, a study published on serological immunity to diphtheria and tetanus in the United States from sera collected during the Third National Health and Nutrition Examination Survey (NHANES III) conducted between 1988 and 1994 determined that, by age 70 years, only $30 \%$ of those tested had serological immunity to either disease (2). These reports provide the impetus to consider how well we are doing with adult immunization in Canada.

The National Advisory Committee on Immunization in Canada recommends that all adults receive tetanus and diphtheria toxoid $(\mathrm{Td})$ vaccines either every 10 years or once in midlife, and that susceptible adults receive measles, mumps, rubella and varicella vaccines $(3,4)$. Recent Canadian guidelines for influenza prevention are that all adults age 65 years and older, and those younger than 65 years who are at high risk for influenza-related complications receive the influenza vaccine on an annual basis (5). Similar indications are in place for pneumococcal vaccines (3). Other immunizations, such as those for hepatitis A, hepatitis B or pertussis may be warranted based on a person's occupational, travel or lifestyle factors (3). In the present article, issues related to pneumococcal, tetanus and diphtheria immunization in adults are reviewed.
\end{abstract}

\section{EPIDEMIOLOGY AND PREVENTION OF PNEUMOCOCCAL DISEASE}

Streptococcus pneumoniae is the most commonly identified bacterial cause of meningitis, otitis media (OM) and community-acquired pneumonia (CAP), and is a frequent cause of bacteremia (6-9). The epidemiology of pneumococcal infection may be examined from the perspectives of clinical manifestations, serotypes and resistance.

S pneumoniae typically colonizes the nasopharynx. While colonization itself does not cause illness, it predisposes the carrier and his or her close contacts to infection.

Colonization rates are high in children - 44\% in those aged six years, $60 \%$ to $80 \%$ in those attending daycare, and greater than $70 \%$ in children younger than three years of age with acute $\mathrm{OM}(10)$. Rates are much lower in healthy adults, in the range of $5 \%$ to $10 \%$ (11). Up to $53 \%$ of colonizing strains in children in the United States are penicillin nonsusceptible (10).

When local defences are evaded, $S$ pneumoniae can cause diseases such as $\mathrm{OM}$, sinusitis and pneumonia. S pneumoniae is the cause of $25 \%$ to $50 \%$ of CAP in adults, causing approximately one case/400 to 500 persons/year and 40,000 deaths/year in the United States $(12,13)$. Pneumococcal disease thus accounts for more deaths than any other vaccine-preventable bacterial disease (12).

Invasive pneumococcal disease has only recently been made reportable in Canada, and in 2000, there were 819 episodes reported to Health Canada (14). The incidence of invasive pneumococcal disease varies from country to country $(8,15-18)$ and sometimes within countries (Table 1$)$. However, the results of surveillance studies are consistent in demonstrating a bimodal distribution, with most disease at the extremes of age - younger than two years and older than 65 years. In 1996, the Sentinel Health Unit Surveillance System, a network of nine health units across Canada, conducted laboratory-based surveillance for invasive pneumococcal infections. From January 1 to December 31, 1996, the incidence of invasive pneumococcal disease among participating areas was 15.1 cases/100,000 population, $55.3 / 100,000$ children younger than five years of age,

${ }^{1}$ Queen Elizabeth II Health Sciences Centre and Dalhousie University, Halifax, Nova Scotia; ${ }^{2}$ Departments of Pathology and Laboratory Medicine, Medicine, and Microbiology $\mathcal{E}$ Infectious Diseases, University of Calgary, Calgary, Alberta

Correspondence and reprints: Dr Lynn Johnston, Room 5014 ACC, Queen Elizabeth II Health Sciences Centre, 1278 Tower Road, Halifax, Nova Scotia B3H 2Y9. Telephone 902-473-8477, fax 902-473-7394, e-mail ljohnsto@is.dal.ca and Dr John Conly, Departments of Pathology and Laboratory Medicine, Medicine, and Microbiology $\mathcal{E}$ Infectious Diseases, Centre for Antimicrobial Resistance, 1638-10th Avenue SW, Calgary, Alberta T3C 0J5. Telephone 403-209-5338, fax 403-209-5347, e-mail jconly@ucalgary.ca 
TABLE 1

Invasive pneumococcal disease in Canada and the United States

\begin{tabular}{lcccccc}
\hline Location & Year & $\begin{array}{c}\text { Incidence } \\
\text { per 100,000 }\end{array}$ & Case fatality (\%) & $\begin{array}{c}\text { Bacteremic } \\
\text { pneumonia (\%) }\end{array}$ & Meningitis (\%) & $\begin{array}{c}\text { Bacteremia } \\
\text { without focus (\%) }\end{array}$ \\
\hline Canada & 1996 & 15.1 & 11.0 & 65.2 & 5.3 & 14.6 \\
Ontario & 1995 & 14.4 & 18.6 & 66.0 & 3.0 & 17.0 \\
Quebec & $1996-1998$ & 16.5 & 7.2 & 61.5 (adults) & 4.1 & 42 (children) \\
United States & 2000 & 21.6 & 10.8 & 59.2 & 5.4 & 30.3 \\
\hline
\end{tabular}

Data from references $15-18$

$46.4 / 100,000$ persons 65 years of age and older, and 80.6 cases $/ 100,000$ persons 85 years of age and older $(9,17)$. The clinical diagnosis was pneumonia in $65.2 \%$, meningitis in $5.3 \%$ and bacteremia in $14.6 \%$ (17). McGeer et al (18) identified a mortality rate of $28 \%$ in adults 65 years of age and older with invasive pneumococcal disease. This is in contrast to children in whom the case fatality rate is $2 \%$, and higher in infants younger than six months of age $(4.3 \%)$ and those with underlying disease (3.8\%) (19).

A number of medical conditions have been identified as predisposing people to invasive pneumococcal disease or poor outcomes from it. They include chronic cardiovascular diseases, chronic pulmonary diseases (excluding asthma), chronic liver disease, diabetes mellitus, asplenia and immunosuppressive conditions related to disease or medications. These medical conditions are the indications for pneumococcal immunization in those younger than 65 years of age (3). An Ontario study (18) found that $77 \%$ of adults with invasive infection had predisposing medical conditions and $93 \%$ of deaths occurred in vaccine-eligible patients. Data in the United States have revealed that blacks, Alaskan natives and certain native Americans are also at increased risk for invasive pneumococcal disease $(12,20)$. This risk appears to be independent of socioeconomic status and underlying diseases (20). In the absence of Canadian data to support or refute these findings, it is not unreasonable to speculate that the same increased risk may exist for Canadian native groups and blacks. In a study involving patients in Canada and the United States, smoking was identified as a risk factor for invasive pneumococal disease (21).

The available literature on serotype distribution, most of it from developed countries, has recently been summarized $(22,23)$. While there are regional variations in the serotypes causing disease, there are also similarities. There is greater serotype variability in adults, who differ from children in serotype distribution. In Canada, the sevenvalent conjugate vaccine contains the serotypes responsible for approximately $85 \%$ of invasive disease in children (22). The match between vaccine and infection strains is not as high in older children (63.6\%) and children with underlying illness (72.9\%) (19). On the other hand, it covers only approximately $60 \%$ of invasive strains in adults (22). Overall, the 23-valent polysaccharide vaccine covers $92 \%$ to $94 \%$ of invasive strains in Canada, including $87.5 \%$ of strains in children $(7,9,19)$. Thus, a relatively small proportion of the known 90 serotypes causes invasive disease and most, but not all, of those are covered by both the seven-valent conjugate and 23-valent polysaccharide vaccines.

Antibiotic-resistant pneumococci have become a worldwide concern. In some European countries, penicillin resistance (intermediate- and high-level) in pneumococci exceeds 40\% (8). From 1995 to 1998 in the United States, penicillin resistance increased from $21 \%$ to $25 \%$ (14\% high-level and $10 \%$ intermediate-level), with $14 \%$ of pneumococci resistant to more than three classes of drugs and more than 33\% resistant to at least one (6). Resistance has not been seen to the same extent in Canada. From 1992 to 2000 , nonsusceptible strains rose from $7.8 \%$ to $15 \%(7,24)$. Almost $4 \%$ of strains were resistant to multiple antibiotics (7). For the year ending March 2002, the National Centre for Streptococcus reported that $7.9 \%$ of isolates from invasive disease in adults were penicillin nonsusceptible (25). Other Canadian studies have confirmed the same level of resistance $(15,18,26)$. Over three years (1996 to 1998), $11 \%$ of isolates causing invasive disease in Quebec were nonsusceptible to penicillin, $4.7 \%$ demonstrating intermediate resistance and 7\% high-level resistance (15). In an Ontario study (18), 6.2\% of isolates from invasive disease in adults were penicillin nonsusceptible in 1995 and 12.7\% in 1997. A study of 154 isolates from across Canada, collected in 1994, found that $9.7 \%$ had reduced susceptibility to penicillin (1.3\% high-level) (7). This same study determined that $85.7 \%$ of intermediate and all resistant strains were covered by the 23 -valent polysaccharide vaccine, but only $59.2 \%$ of intermediate strains were covered by the seven-valent conjugate vaccine (7). Another cross-Canada survey of isolates from invasive disease in the mid-1990s reported $8.4 \%$ of strains with intermediate-level resistance and $3.3 \%$ with high-level resistance (26). Scheifele et al (19) found that $95.4 \%$ of resistant strains causing invasive disease in Canadian children were serotypes contained in the seven-valent vaccine (19). The emergence of antimicrobial resistance has given new importance to preventing pneumococcal disease with immunization.

The 23-valent polysaccharide and seven-valent conjugate pneumococcal vaccines are licensed for use in Canada for different indications. The serotypes chosen for vaccines are those most commonly responsible for disease. Protection from invasive infection relies largely on the development of antibodies against the capsular antigens, related to the particular strain (some being more immuno- 
genic than others) and immune function of the host. Children younger than two years of age have not developed the T-cell independent immunity required to respond to polysaccharide vaccines (27). Conjugation to T-celldependent proteins makes the seven-valent vaccine much more immunogenic in young children who have T-celldependent responses soon after birth (27). Other individuals may have congenital or acquired defects affecting vaccine response. It is not clear what level of antibody provides protection, and it may be that protective levels vary for different serotypes and disease manifestations (12). All these factors must be considered when evaluating immunogenicity and, ultimately, efficacy. The 23-valent polysaccharide vaccine results in an antibody response in $80 \%$ of healthy adults and otherwise healthy asplenic individuals (12). Antibody levels in most immunocompetent older adults are similar to those in younger individuals (28). Responses may be lower in the elderly and those who have alcoholic cirrhosis, chronic obstructive pulmonary disease and insulin-dependent diabetes, and especially in those who are at greatest risk for disease and morbidity, including individuals with hematological malignancy, renal failure, nephrotic syndrome and immunodeficient states (12). Antibody levels persist for at least five years, possibly longer, in healthy adults but for lesser periods in those who initially respond less well to vaccination $(12,13,28)$. There is insufficient information to reach conclusions regarding the response of healthy or immunocompromised older children and adults to the seven-valent conjugate vaccine. Even if improved immune responsiveness is noted, the poor coverage offered by the seven-valent conjugate vaccine for serotypes causing disease in adults is a significant drawback. The seven-valent conjugate vaccine cannot be recommended for use in adults.

There has been much debate over the years about the efficacy of the 23-valent polysaccharide vaccine. Studies have not conclusively shown benefit in the prevention of nonbacteremic pneumonia in adults $(12,28)$. Several casecontrol and cohort studies have demonstrated an approximate $50 \%$ to $80 \%$ effectiveness ( $75 \%$ in immunocompetent elderly persons) in preventing invasive disease $(13,28)$. A meta-analysis of randomized clinical trials using primarily a 14-valent polysaccharide vaccine showed efficacy in preventing bacteremic and definitive pneumococcal pneumonia in low risk individuals (29). There have been insufficient data collected to determine efficacy in immunocompromised individuals (as opposed to those with chronic but not immunocompromising disease), where it is expected to be lower. A recent study of the cost-effectiveness of vaccination with the 23 -valent polysaccharide vaccine against pneumococcal bacteremia in the elderly found that, under the usual (and even reasonable worst case) assumptions of cost and efficacy, this strategy is actually cost saving (28). A Quebec study (30) estimated that the cost of a public pneumococcal vaccine program is approximately $\$ 5.80 /$ dose, which is comparable with other services provided for individuals with chronic illnesses (30).
TETANUS EPIDEMIOLOGY AND PREVENTION

Although conditions that allow for tetanus remain, the disease is very rare in the developed world, due in large part to the widespread use of TT-containing vaccines and postexposure wound care. Between two and seven tetanus cases per year occur in England and Wales, primarily in older people (mainly women) who have never been vaccinated (1). In Canada, one case of tetanus was reported in 1999, two in 2000, and four as of September 30, 2001 (14). The Unites States reported 36 to 51 cases/year between 1992 and 1999 , a rate of 0.15 cases $/ 1,000,000$ population $(31,32)$. The epidemiology of tetanus cases reported in the United States between 1995 and 1997 has been described in some detail (32). Although only 35\% of cases occurred in adults 60 years of age and older, older adults had a nearly sevenfold greater risk for tetanus than children and adolescents. They also experienced a higher case fatality rate. Men predominated among patients aged 20 to 59 years and women among those 60 years of age and older. Only 13\% of patients reported having received a primary series of TT in the past. Sixty-one per cent of patients seeking care for their injuries did not receive appropriate tetanus prophylaxis. Eleven per cent of cases were in injection drug users who had no known injuries. The disproportionate number of cases in the 20 to 59 years of age group was in part related to the increase in tetanus among injection drug users. Three cases were related to elective surgery. The overall case-fatality ratio for the 124 cases was $11 \%$, with no deaths among the 16 patients who had previously received at least three doses of TT. The rate was $6 \%$ for patients who had received one to two doses and $15 \%$ for unvaccinated individuals. Thus, any level of immunization appears to offer some protection.

This low rate of tetanus exists even though a significant proportion of the older population appears to be susceptible. As noted, the NHANES III survey found that only $50 \%$ of adults aged 60 years and older were tetanus immune (2). At every age, fewer women than men have protective levels of antibody and the discrepancy increases with age such that at 70 years, $45 \%$ of men but only $21 \%$ of women are protected (2). It is not clear how many of those with no detectable antibody have previously been fully or partially immunized, or not immunized at all. Although under-reporting contributes somewhat to the low frequency of tetanus, presumably improved wound care (including specific tetanus prophylaxis), less exposure to tetanus spores in a more urban population, and clinically important, albeit serologically undetectable, antibody levels play a role. While a low rate of infection tends to give a sense of assurance that current strategies have been successful, can we justify even one case of an easily preventable disease? On the other hand, is it cost effective to mount a rigorous adult tetanus immunization program for such a rare disease? Under a variety of cost and disease rate assumptions, Balestra and Littenberg (33) determined that a strategy of immunizing all 65-year-olds is cost effective compared with two alternate strategies: a booster 
every 10 years, or no intervention after age six years, except for wound prophylaxis (33). The Canadian Immunization Guide gives two options for maintaining tetanus immunity. It suggests administering booster doses at 10 yearly intervals or reviewing immunization status at least once during adult life and giving a dose of $\mathrm{Td}$ to everyone who has not had one within the previous 10 years (3). A primary immunization series is required by those who have never received it.

\section{DIPHTHERIA EPIDEMIOLOGY AND IMMUNITY}

Diphtheria is even less common than tetanus in North America. In Canada, only two to five cases were reported annually from 1986 to 1995 (34). Since then, there has been only one case every one to two years (14). However, it has been noted that toxigenic strains continue to be isolated in carriers, mainly in northern and western Canada and among aboriginal populations (34). From 1980 to 1995 , a total of 41 respiratory diphtheria cases were reported in the United States, all in unvaccinated children (35). Two of the cases in the United States were imported from the new independent states of the former Soviet Union during their protracted outbreak (36). Surveillance cultures related to a case of diphtheria in 1996 detected six carriers (5\%) among 133 that were cultured, four with toxigenic strains (35). Four of the six were children, all of whom were fully or partially immunized (35). Similar to tetanus, evidence suggests that the severity of disease decreases with the number of $\mathrm{Td}$ doses received, and deaths do not occur in individuals who have been fully immunized (37).

The NHANES III survey found that $60.5 \%$ of participants were diphtheria-immune (2). Rates varied, however, from a high of $91 \%$ in those aged six to 11 years to $29.5 \%$ in those older than 70 years of age. While discrepancies existed between men and women, they were not as pronounced as those for tetanus immunity (2). In 1996, the Division of Immunization, Health Canada conducted a serosurvey for diphtheria antibody among donors in five blood collection centres. The proportion of susceptible donors ranged from $10 \%$ in those 30 to 39 years of age to $36 \%$ in those 60 years of age or older (34).

The resurgence of diphtheria in the former Soviet Union during the 1990s highlights the potential for its occurrence in developed countries given the appropriate set of circumstances. A number of factors are considered to have contributed to that epidemic (38). Beginning in the 1980s, the school entry booster was dropped, more contradictions to vaccination were allowed, and an anti-immunization movement gained a foothold. Childhood immunization rates in the Russian Federation fell as low as $70 \%$. Additionally, many children received vaccines with low antigen content. Military recruits, not routinely immunized against diphtheria until 1990, may have introduced new strains into the general population. Because of the previously successful Russian childhood immunization program with resultant lowered opportunity for natural boosting in adults and wan- ing of vaccine-induced immunity in the absence of booster doses, there was a large susceptible adult population. By itself, a large number of susceptible people does not usually result in epidemic diphtheria. However, poor social circumstances associated with this era in Soviet history may have resulted in poor hygiene and crowded conditions that enhanced the spread of infection (38). This convergence of social and political factors leading to disease is not unique to diphtheria, but characterizes the spread of other new and re-emerging infections.

It is reassuring to note that the epidemic was controlled by vaccination efforts in adults and children (38). Although the likelihood of a diphtheria epidemic in Canada is remote, given that asymptomatic carriers exist (35) and the low level of immunity in older adults $(2,34)$, the potential for isolated cases certainly exists. Focusing efforts on ensuring primary immunization in childhood with a single booster at midlife should prevent diphtheria as well as tetanus.

\section{IMPROVING ADULT IMMUNIZATION}

Considerable data show that immunization rates in adults against diphtheria, tetanus and pneumococcal disease are suboptimal. A 1994 national survey of vaccine coverage in noninstitutionalized Canadians showed that 3\% of those 65 years of age and older and $9 \%$ of those 18 to 24 years of age had received $\mathrm{Td}$ within the past 10 years (34). The pneumococcal vaccine rate was $1 \%$ in adults aged 65 years and older (34). A 1996 telephone survey of noninstitutionalized adults in Quebec found that among those 65 years of age and older, $10 \%$ had received TT and $1.8 \%$ had received the pneumococcal vaccine (39). On a more positive note, a 2001 telephone survey found that $42.4 \%$ of adults aged 65 years and older and $15.4 \%$ aged 18 to 64 years with high risk medical conditions had ever received the pneumococcal vaccine (40). A similar pattern has been seen in the United States. Pneumococcal immunization rates improved from 28\% to 46\% between 1993 and 1997 among adults aged 65 years and older, but remained considerably lower among adults aged 18 to 49 years (12\%) and 50 to 64 years (20\%) with chronic conditions (41). In the 65 years of age and older group, vaccination levels are affected by race and/or ethnicity, poverty status, education level and the number of physician contacts during the previous year (41). This is of concern given the racial differences in risk for invasive pneumococcal disease.

Why are adult immunization rates so poor? A number of reasons have been suggested by the United States National Vaccine Advisory Committee. They include misperceptions about the risks of vaccine-preventable diseases in adults, vaccine safety and efficacy, the size of the target population, the lack of regulatory and/or legal requirements, reimbursement and the lack of coordinated adult immunization programs (42). One study found that most adults eligible for pneumococcal vaccines who visited a physician during the previous year did not receive it (41). The Quebec survey (39) found that 75\% of those vaccinated 
properly for influenza during the year were not up-to-date with their tetanus immunizations. Clearly, there are missed immunization opportunities in the physician's office. In a 1996 survey of primary care physicians in Massachusetts, $24 \%$ of respondents identified oversight as the most important barrier to reducing the number of patients they immunize (43). Unfortunately, hospitalization frequently represents a missed opportunity for vaccination. It has been observed that only $19.6 \%$ of hospitalized adults 65 years of age or older received or were noted to have had pneumococcal immunization during that hospitalization (44).

There is a large body of research on strategies to improve preventive strategies in health care, including vaccination rates. In 1999, Shefer et al (45) published a systematic review of interventions to improve immunization. Interventions were classified into four broad groupings: those to increase demand (including community education, patient reminders and incentives, and patient-held medical records), those to enhance access (such as decreasing vaccine costs to families and providing services in nonmedical settings), legal or regulatory interventions, and providerbased interventions (including provider education, feedback and reminders). Not surprisingly, the evidence supports a benefit to legal and regulatory interventions and those that enhance access. For interventions to increase community demand, multicomponent strategies are required for success. There is little evidence to support that provider education increases vaccine uptake. Patient and provider reminder (vaccine due) and/or recall (missed vaccine) systems both have evidence to support efficacy. Two other studies have confirmed the benefit of patient reminders and recalls to improve vaccine delivery $(46,47)$. A meta-analysis of interventions to increase the use of adult immunization and cancer screening services found that the strongest intervention involved organizational change characterized by such elements as the use of separate clinics devoted to prevention, the use of a planned care visit for prevention, or the designation of nonphysician staff to do specific prevention activities (47). Because of different

\section{REFERENCES}

1. Public Health Laboratory Service. Two recent cases of severe tetanus. Commun Dis Rep CDR Wkly 2002;12:17.

2. McQuillan GM, Kruszon-Moran D, Deforest A, Chu SY, Wharton M. Serologic immunity to diphtheria and tetanus in the United States. Ann Intern Med 2002;136:660-6.

3. National Advisory Committee on Immunization. Recommended immunization for infants, children and adults. In: Canadian Immunization Guide, 5th edn. Ottawa: Health Canada, 1998:45-60.

4. National Advisory Committee on Immunization. Statement on recommended use of varicella virus vaccine. Can Commun Dis Rep 1999;25(ACS-1):1-16.

5. National Advisory Committee on Immunization. Statement on influenza vaccination for the 1999-2000 season. Can Commun Dis Rep 1999;25(ACS-2):1-16.

6. Whitney CG, Farley MM, Hadler J, et al. Increasing prevalence of multidrug-resistant Streptococcus pneumoniae in the United States. N Engl J Med 2000;343:1917-24.

7. Lovgren M, Spika JS, Talbot JA. Invasive Streptococcus pneumoniae infections: Serotype distribution and antimicrobial resistance in Canada, 1992-1995. CMAJ 1998;158:327-31. types of strategies used in various studies, estimates of cost effectiveness cannot be made from meta-analysis (46) and have not been examined in individual studies (45).

Factors that have been suggested as contributing to the success of the United States pneumococcal immunization program are the development of standards for adult immunization practice, the establishment of a national adult immunization week, the publication of a comprehensive strategy to improve adult vaccination by the Department of Health and Human Services, and enhanced state and local programs (41). The United States Task Force on Community Preventive Services recommended that a starting point for addressing vaccine-preventable disease problems would be to assess activities that are already underway, current levels of vaccination coverage and disease rates, and compare them with national and local goals (48). Particular attention should be given to underserved populations at highest risk for disease. There are national goals for pneumococcal immunization (9). Canada needs clearly articulated standards for $\mathrm{Td}$ immunization, more Canadian data on adult vaccination rates and consumer barriers to immunization, and well-defined adult immunization programs.

Adult infectious disease physicians may see little role for themselves in participating in adult immunization services. However, the objectives of training in infectious diseases include the application of the principles of communicable disease epidemiology to their prevention in the community. The Infectious Disease Society of America has adopted practice standards related to immunization. Two of the standards are that providers use all clinical encounters to screen and, when indicated, immunize patients, and that they conduct regular audits to assess immunization coverage levels in the population that they serve (49). Our training should leave us well placed to work with local and provincial health officials to develop goals and strategies for adult immunization programs and evaluate their success. It behooves the Canadian Infectious Disease Society to take a leadership role in this area and formalize its position on adult immunization.

8. Gaillat J. Epidemiology of systemic pneumococcal infections in western Europe. Can J Infect Dis 1999;10(Suppl A):s35-40.

9. Health Canada. Preventing pneumococcal disease: A Canadian consensus conference - 16-18 February, 1998. Can Commun Dis Rep 1999;25:25-35.

10. Giebink GS. The prevention of pneumococcal disease in children. N Engl J Med 2001;345:1177-83.

11. Musher DM. Streptococcus pneumoniae. In Mandell GL, Bennett JE, Dolin R, eds. Principles and Practice of Infectious Diseases. Philadelphia: Churchill Livingstone, 2000:2128-47.

12. Prevention of pneumococcal disease: Recommendations of the Advisory Committee on Immunization Practices (ACIP). MMWR Recomm Rep 1997;46(RR-8):1-24.

13. Fedson DS, Shapiro ED, LaForce FM, et al. Pneumococcal vaccine after 15 years of use: Another view. Arch Intern Med 1994;154:2531-5.

14. Health Canada. Notifiable diseases summary. Can Commun Dis Rep 2002;28:50-1.

15. Jette LP, Delage G, Ringuette L, et al. Surveillance of invasive Streptococcus pneumoniae infection in the province of Quebec, 
Canada, from 1996 to 1998: Serotype distribution, antimicrobial susceptibility, and clinical characteristics. J Clin Microbiol 2001;39:733-7.

16. Active Bacterial Core Surveillance (ABCs) Report Emerging Infections Program Network Streptococcus pneumoniae, 2000. <http://www.cdc.gov/ncidod/dbmd/abcs/survreports/spneu00.pdf> Version current at June 5, 2002

17. Kertesz DA, Senzilet L, Alagaratnam M, Lovgren M, Talbot JA, Spika JS. Invasive pneumococcal disease in Canada 1996: Results from Sentinel Health Unit Surveillance System. Can J Infect Dis 1999;10(Suppl A):s22-23.

18. McGeer A, Green K, Landry L, Talbot J, Goldenberg E. Assessing the potential impact of vaccination programs on invasive pneumococcal disease: Data from population-based surveillance. Can J Infect Dis 1999;10(Suppl A):s24-26.

19. Scheifele D, Halperin S, Pelletier L, Talbot J. Invasive pneumococcal infections in Canadian children, 1991-1998: Implications for new vaccination strategies. Clin Infect Dis 2000;31:58-64

20. Whitney CG, Schaffner W, Butler JC. Rethinking recommendations for use of pneumococal vaccines in adults. Clin Infect Dis 2001;33:662-75.

21. Nuorti JP, Butler JC, Farley MM, et al. Cigarette smoking and invasive pneumococcal disease. N Engl J Med 2000;342:681-9.

22. Hausdorff WP, Bryant J, Paradiso PR, Siber GR. Which pneumococcal serogroups cause the most invasive disease: Implications for conjugate vaccine formulation and use, Part I. Clin Infect Dis 2000;30:100-21.

23. Hausdorff WP, Bryant J, Kloek C, Paradiso PR, Siber GR. The contribution of specific pneumococcal serogroups to different disease manifestations: Implications for conjugate vaccine formulation and use, Part II. Clin Infect Dis 2000;30:122-40.

24. Lovgren M, Talbot JA. Antimicrobial-resistant Streptococcus pneumoniae. Can J Infect Dis 1999;10(Suppl A):27-29.

25. National Centre for Streptococcus - Annual Reports. <http://www2.provlab.ab.ca/vlab/ncs/ar2002.pdf > Version current at June 4, 2002

26. Simor A, Louie M, Low DE. Canadian national survey of prevalence of antimicrobial resistance among clinical isolates of Streptococcus pneumoniae. Antimicrob Agents Chemother 1996;40:2190-3.

27. Preventing pneumococcal disease among infants and young children: Recommendations of the Advisory Committee on Immunization Practices (ACIP). MMWR Recomm Rep 2000;49(RR-9):1-35.

28. Sisk JE, Moskowitz AJ, Whang W, et al. Cost-effectiveness of vaccination against pneumococcal bacteremia among elderly people. JAMA 1997;278:1333-9.

29. Fine MJ, Smith MA, Carson CA. Efficacy of pneumococcal vaccine in adults: A meta-analysis of randomized controlled trials. Arch Intern Med 1994;154:2666-77.

30. DeWals PD, Guay M, Drapeau J, et al. Pneumococcal immunization program: Cost-utility analysis for Quebec. Can J Infect Dis 1999;10(Suppl A):s46-47.
31. Summary of notifiable diseases, United States, 1998. MMWR Morb Mortal Wkly Rep 1999;47:1-92.

32. Tetanus surveillance - United States, 1995-1997. MMWR Morb Mortal Wkly Rep 1998;47(SS-2):1-13.

33. Balestra DJ, Littenberg B. Should adult tetanus immunization be given as a single vaccination at age 65 ? A cost-effectiveness analysis. J Gen Intern Med 1993;8:405-12.

34. Health Canada. Epidemiology of selected vaccine-preventable diseases. Can Commun Dis Rep 1997;23:S4.

35. Toxigenic Corynebacterium diphtheriae - Northern Plains Indian community. MMWR Morb Mortal Wkly Rep 1997;46:506-10.

36. Respiratory diphtheria caused by Corynebacterium ulcerans Terre Haute, Indiana, 1996. MMWR Morb Mortal Wkly Rep 1997;46:330-2.

37. Gardner P. Issues related to the decennial tetanus-diphtheria toxoid booster recommendations in adults. Infect Dis Clin North Am 2001;15:143-51.

38. Vitek CR, Wharton M. Diphtheria in the former Soviet Union: Reemergence of a pandemic disease. Emerg Infect Dis 1998;4:539-49.

39. Duclos P, Arruda H, Dessau J-C, et al. Immunization survey of noninstitutionalized adults - Quebec (as of May 30, 1996). Can Commun Dis Rep 1996;22:21.

40. Squires SG, Macey JF, Tam T. Progress towards Canadian target coverage rates for influenza and pneumococcal immunizations. Can Commun Dis Rep 2001;27:90-1.

41. Influenza, pneumococcal, and tetanus toxoid vaccination of adults - United States, 1993-1997. MMWR Morb Mortal Wkly Rep 2000;49:39-52.

42. Fedson DS, for the National Vaccine Advisory Committee. Adult immunization. Summary of the National Vaccine Advisory Committee Report. JAMA 1994;272:1133-7.

43. Noe CA, Markson LJ. Pneumococcal vaccination: Perceptions of primary care physicians. Prev Med 1998;27:767-72.

44. Missed opportunities for pneumococcal and influenza vaccination of medicare pneumonia inpatients - 12 Western states, 1995. MMWR Morb Mortal Wkly Rep 1997;46:919-23.

45. Shefer A, Briss P, Rodewald L, et al. Improving immunization coverage rates: An evidence-based review of the literature. Epidemiol Rev 1999;21:96-142.

46. Szilagyi PG, Bordley C, Vann JC, et al. Effect of patient reminder/recall interventions on immunization rates. JAMA 2000;284:1820-7.

47. Stone EG, Morton SC, Hulscher ME, et al. Interventions that increase use of adult immunization and cancer screening services: A meta-analysis. Ann Intern Med 2002;136:641-51.

48. Task Force on Community Preventive Services. Recommendations regarding interventions to improve vaccination coverage in children, adolescents, and adults. Am J Prev Med 2000;18(Suppl 1):92-6.

49. Gershon AA, Gardner P, Peter G, Nichols K, Orenstein W. Quality standards for immunization. Clin Infect Dis 1997;25:782-6. 


\section{Erratum}

In the Adult Infectious Disease Note "Routine adult immunization in Canada: Recommendations and performance" (Can J Infect Dis 2002;13(4):226-231), references 39 and 40 were cited incorrectly. The references, as they should have appeared, are printed below.

39. Duclos P, Arruda H, Dessau J-C, et al. Immunization survey of non-institutionalized adults - Quebec (as of May 30, 1996). Can Commun Dis Rep 1996;22:21.

40. Squires SG, Macey JF, Tam T. Progress towards Canadian target coverage rates for influenza and pneumococcal immunizations. Can Commun Dis Rep 2001;27:90-1. 


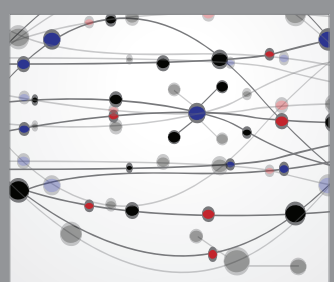

The Scientific World Journal
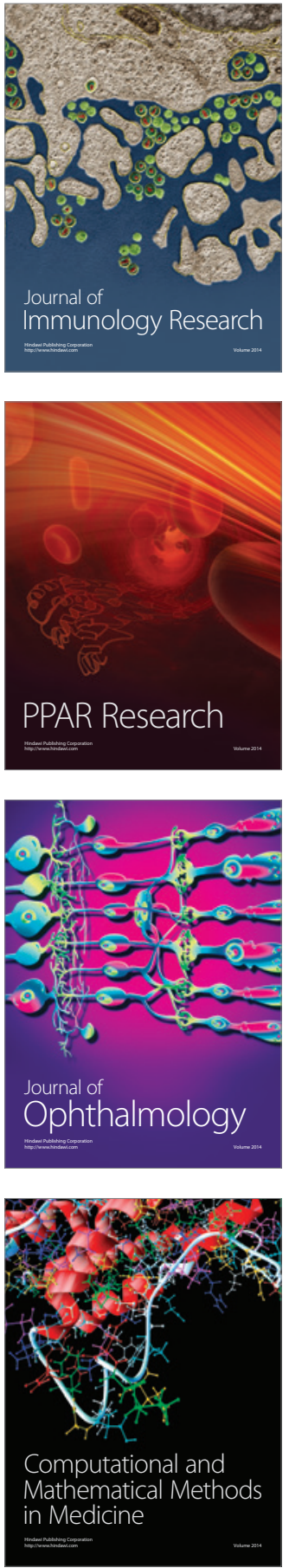

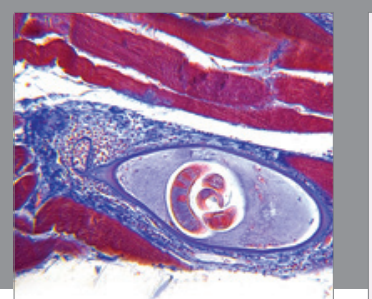

Gastroenterology Research and Practice

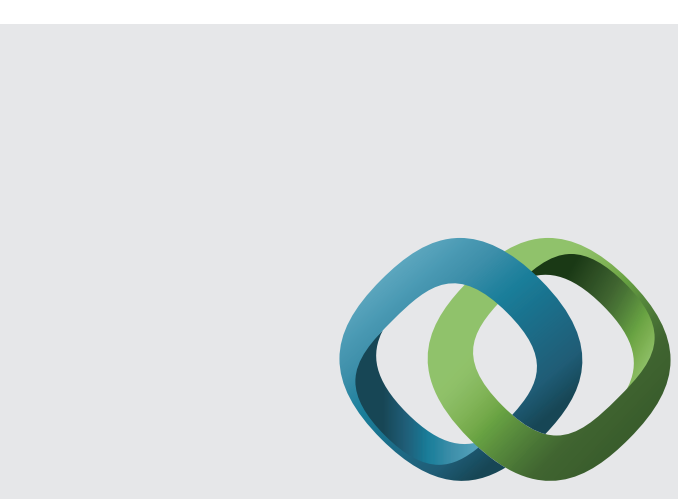

\section{Hindawi}

Submit your manuscripts at

http://www.hindawi.com
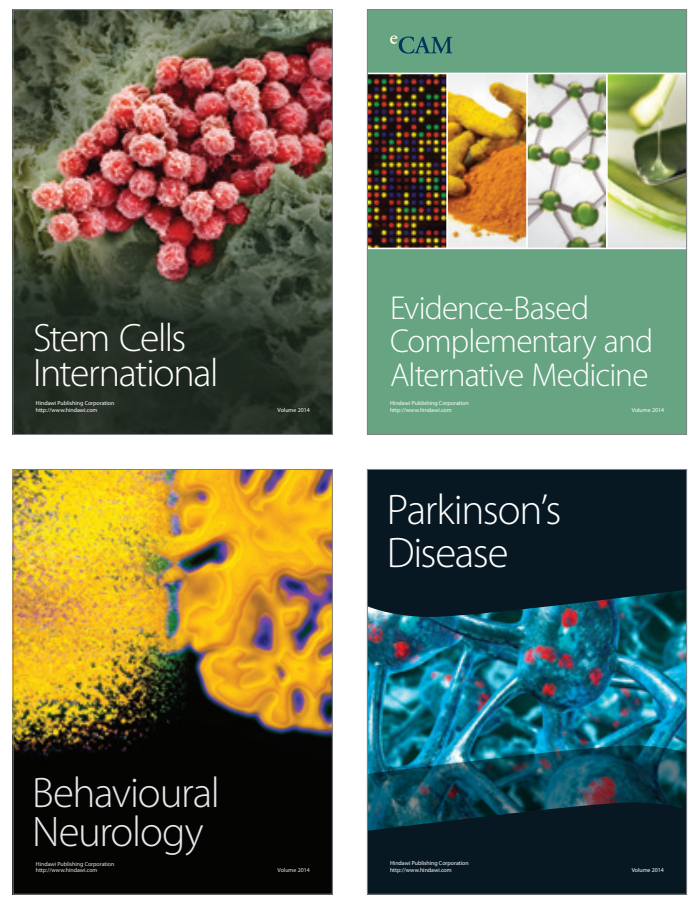
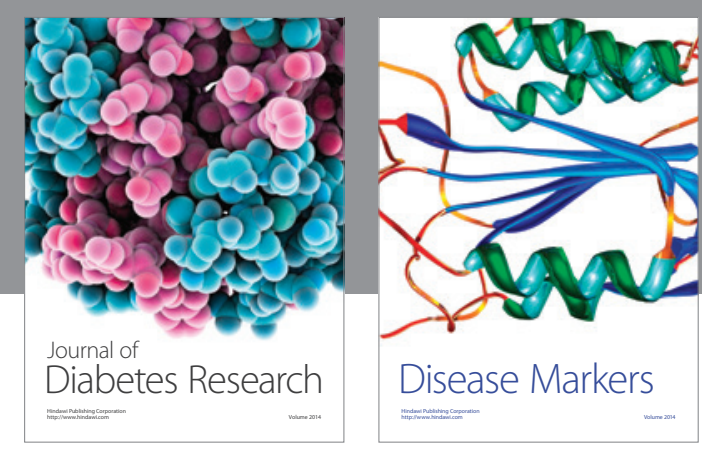

Disease Markers
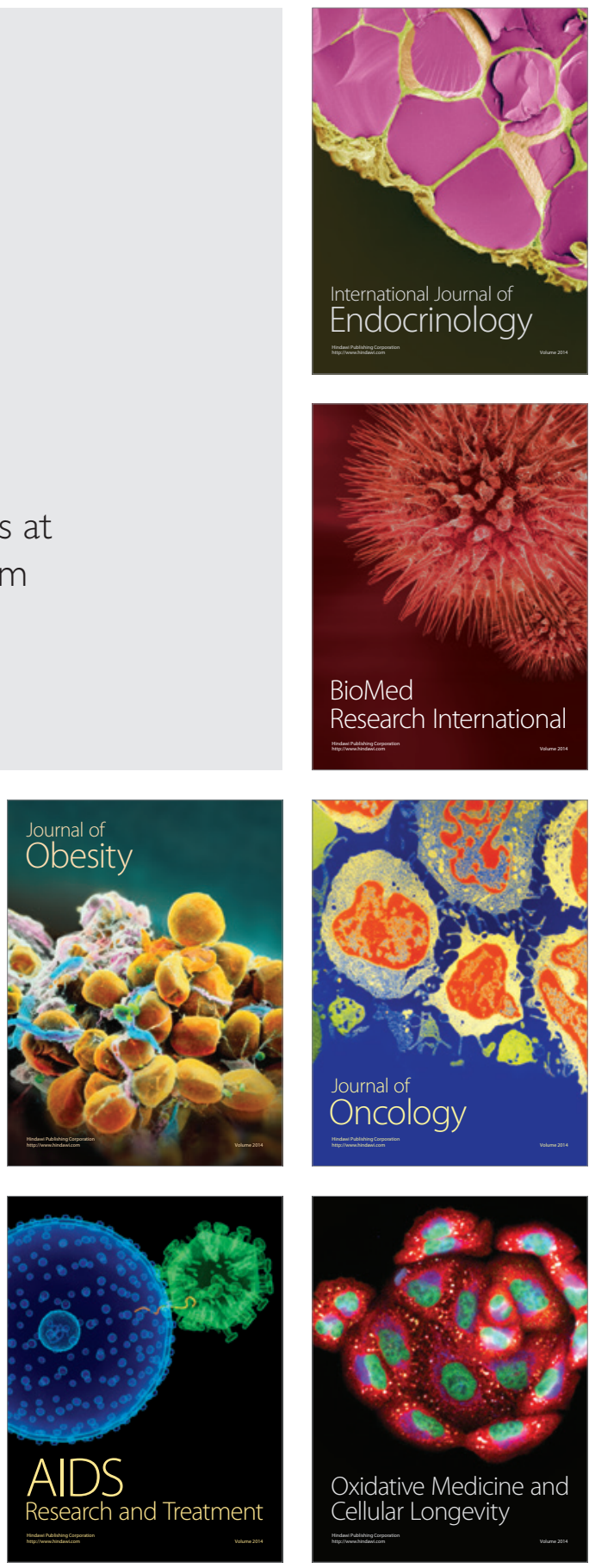